\title{
An ENU-induced mutation in the mouse $R n f 212$ gene is associated with male meiotic failure and infertility
}

\author{
Yasuhiro Fujiwara ${ }^{1,2,}{ }^{*}$, Hirokazu Matsumoto ${ }^{3,}{ }^{,}$, Kouyou Akiyama ${ }^{3}$, Anuj Srivastava ${ }^{2}$, \\ Mizuho Chikushi ${ }^{3}$, Mary Ann Handel $^{2}$ and Tetsuo Kunieda ${ }^{3}$ \\ ${ }^{1}$ Graduate School of Natural Science and Technology, Okayama University, 1-1-1 Tsushima-naka, Kita-ku, \\ Okayama 700-8530, Japan, ${ }^{2}$ The Jackson Laboratory, 600 Main Street, Bar Harbor, Maine 04609, USA and \\ ${ }^{3}$ Graduate School of Environmental and Life Science, Okayama University, 1-1-1 Tsushima-naka, Kita-ku, \\ Okayama 700-8530, Japan
}

Correspondence should be addressed to T Kunieda; Email: tkunieda@cc.okayama-u.ac.jp

*(Y Fujiwara and $\mathrm{H}$ Matsumoto contributed equally to this work)

\begin{abstract}
The ENU-induced repro57 mutation was identified in an unbiased screen for the discovery of novel genes for fertility. Male repro57 homozygous mice are infertile and exhibit significantly reduced testis weight compared with WT mice. Histological examination of mutant testes revealed that spermatocytes degenerated during late prophase, and no mature spermatozoa were found in the seminiferous epithelium, suggesting that infertility is caused by the arrest of spermatogenesis at late meiotic prophase. Consistent with this hypothesis, the number of foci with MLH1, a protein essential for crossing over, is greatly reduced in repro57 mutant spermatocytes, which also lack chiasmata between homologs and exhibit premature dissociation of XY chromosomes. In repro57 mutant mice, we identified a mutation in the Rnf212 gene, encoding Ring finger protein 212. The overall phenotype of repro57 mice is consistent with the recently reported phenotype of the Rnf212 knockout mice; slight differences may be due to genetic background effects. Thus, the repro57 nonsense mutation provides a new allele of the mouse Rnf212 gene.

Reproduction (2015) 149 67-74
\end{abstract}

\section{Introduction}

During meiosis, homologous chromosomes undergo highly programed and evolutionarily conserved processes, including pairing of homologous chromosomes, synapsis, recombination to form chiasmata between homologs, and chromosome segregation mediated by chiasmata resolution. At the onset of meiosis, recombination is initiated by formation of DNA double-strand breaks (DSBs) by SPO11 in most organisms (Phadnis et al. 2011). DNA ends at the DSB sites are loaded with the recombinases RAD51 and DMC1, promoting homolog interaction leading to synapsis, which is mediated by the formation of a unique structural scaffold, the synaptonemal complex (SC) (Cromie \& Smith 2007). During recombination, DSBs are repaired by either crossing over (CO) or non-CO process. CO sites are created by exchange of non-sister chromatids between homologs, and formation of at least one $\mathrm{CO}$ on each chromosome is essential for the accurate segregation of homologous chromosomes (Baudat et al. 2013). However, the precise mechanisms and full repertoire of genes responsible for mammalian CO recombination are still unclear (Handel \& Schimenti
2010, Bolcun-Filas \& Schimenti 2012). ring finger protein 212 (Rnf212) gene has been shown to be essential for meiotic recombination in Caenorhabditis elegans (Jantsch et al. 2004, Bhalla et al. 2008), and, in humans, RNF212 has been associated with variation in the genome-wide recombination rate (Kong et al. 2008, Chowdhury et al. 2009). Moreover, the infertility and CO failure of mice bearing a targeted knockout of the mouse gene, Rnf212 ${ }^{\text {tm } 1 \text { Nhtr }}$ (herein referred to as Rnf212 KO) provide evidence for an essential role of RNF212 in mammalian CO recombination (Reynolds et al. 2013).

To discover genes involved in meiosis and reproduction, an unbiased phenotype-driven approach using $\mathrm{N}$-ethyl$\mathrm{N}$-nitrosourea (ENU)-induced mutagenesis and fertility screening was conducted by the Reproductive Genomics Program at The Jackson Laboratory (JAX) (Lessard et al. 2004, Handel et al. 2006). From this program, we identified homozygous repro57 mice as infertile and report herein that the male repro57 mutant mice exhibit meiotic arrest with defective CO. The repro57 mice have a nonsense mutation in exon 4 of the Rnf 212 gene, encoding a, RNF212 (also known as Zip3 in budding yeast and ZHP3 in worms), thereby identifying a new allele of Rnf212. 


\section{Materials and methods}

\section{Mice}

The repro57 mice used in this study were produced by the NIHsupported Reproductive Genomics program at JAX (http:// reproductivegenomics.jax.org/), where each mutant line was designated by a 'repro' number in the order of appearance. Mice of the JF1/Ms (JF1) strain were obtained from the National Institute of Genetics (Mishima, Japan), and C3HeB/FeJ $(\mathrm{C} 3 \mathrm{H})$ mice were obtained from CLEA Japan (Tokyo, Japan). The repro57 mutation was induced in a $\mathrm{C} 57 \mathrm{BL} / 6 \mathrm{~J}$ (B6) background and mutagenized mice were subsequently outcrossed to $\mathrm{C} 3 \mathrm{H}$ (Handel et al. 2006), with creation of a repro57-C3H congenic line. For experimental analyses, repro57 homozygous mice were obtained by mating of repro57 heterozygous mice. Mice were killed using $\mathrm{CO}_{2}$ at 10-12 weeks (or at 0-5 weeks of age to follow the first wave of spermatogenesis). All experiments using animals were approved by the animal care and use regulatory committees of both Okayama University and JAX.

\section{Mapping the repro57 mutation}

Adult B6 mice were mutagenized with ENU and the repro57 infertility phenotype was identified in the third generation by mating with normal mice for 3 weeks. More precise phenotypes were subsequently determined from IVF and histological examination of reproductive tissues (Handel et al. 2006). To establish the chromosomal linkage of the repro57 mutation, genome scans using polymorphic microsatellite markers were performed on DNA obtained from affected and unaffected mice. For fine mapping analysis, 740 $\mathrm{F} 2$ mice were produced by mating the F1 mice derived from an intercross between the repro57 and JF1 mice. The F2 offspring were phenotyped by histology and genotyped using polymorphic microsatellite markers to narrow the candidate region.

DNA sequencing was performed to identify the site of the repro57 mutation. Genomic DNA was isolated from the liver of repro57 mutants and founder strain B6 mice. DNA samples were re-extracted once with phenol-chloroform; the 260:280 ratio was $\sim 2.0$. Whole-genome libraries were prepared and subjected to sequencing on the illumina HiSeq 2000 (illumine, CA, USA); sequence analysis was focused on the candidate region identified from mapping.

\section{Bioinformatics analysis}

The dataset was subjected to quality control using NGSQCtoolkit v2.3 and reads with base qualities greater $\geq 30$ over $70 \%$ of total read length were used in the downstream analysis (Patel \& Jain 2012). High-quality reads were mapped to the mouse genome (build-mm10) using BWAv0.5.10-tpx (Li \& Durbin 2009). The resulting alignment was sorted by coordinates and further converted to a binary alignment map (BAM) format by Picardv 1.8.4- SortSam utility (http://picard.sourceforge.net). Picard-MarkDuplicates module was used to remove duplicates from the data. Subsequently, Genome Analysis tool kit (GATK) v2.2-16 (McKenna et al. 2010, DePristo et al. 2011) modules IndelRealigner and BaseRecalibrator were used to pre-process the alignments. The realigned and recalibrated BAM alignment file was used as an input to GATK-UnifiedGenotyper (variant caller) at parameters, -stand_call_conf 50.0, -stand_ emit_conf 30.0 and -dcov 200, and variant calls were restricted to the target region. Finally, variant calls were annotated by snpEffv2.0.5 (Cingolani et al. 2012).

\section{Cytological preparations and immunostaining}

\section{Histology}

Testes from mice were fixed in Bouin's solution and paraffin embedded. Sections $(5 \mu \mathrm{m})$ were stained with hematoxylin and eosin (HE) for evaluation of spermatogenesis. Immunohistochemistry was performed as described previously (Fujiwara et al. 2013). Mouse monoclonal anti-human proliferating cell nuclear antigen (PCNA) primary antibody and anti-rat IgG HRP-labeled secondary antibody (Table 1) were used to label the histological sections. For fluorescence immunostaining, goat polyclonal anti-human DMC1 (Table 1) was used in PBS containing $5 \%$ skim milk for $3 \mathrm{~h}$ at $37^{\circ} \mathrm{C}$. A donkey anti-goat IgG-DyLight 488 secondary antibody

Table 1 Antibodies for fluorescence immunostaining.

\begin{tabular}{|c|c|c|c|}
\hline Antibody & Host & Cat. number or reference & Dilution \\
\hline Anti-ßactin & Rabbit & Abcam, Cambridge, UK, ab8227 & $1: 1000$ \\
\hline Anti-DMC1 & Goat & Santa Cruz, sc-8973 & $1: 50$ \\
\hline Anti- $\gamma \mathrm{H} 2 \mathrm{AX}$ & Mouse & Upstate, Billerica, MA, USA, JBW301 & $1: 500$ \\
\hline Anti-H1T & Guinea pig & Handel Lab, Bar Harbor, ME, USA (Cobb et al. 1999) & $1: 1000$ \\
\hline Anti-MLH1 & Mouse & BD Pharmingen, San Jose, CA, USA, 551091 & $1: 50$ \\
\hline Anti-PCNA & Mouse & Santa Cruz, sc-25280 & $1: 500$ \\
\hline Anti-SYCP1 & Rabbit & Novous Biologicals, Littleton, CO, USA, NB300-229 & $1: 100$ \\
\hline Anti-SYCP3 & Guinea pig & de Massy Lab, Montpellier, France & $1: 1500$ \\
\hline Anti-SYCP3 & Rabbit & Chuma Lab, Kyoto, Japan & $1: 1000$ \\
\hline Anti-RNF212 & Rabbit & Hunter Lab, Davis, CA, USA (Reynolds et al. 2013) & $1: 1000$ \\
\hline Anti-goat IgG-DyLight 488 & Donkey & Jackson Immuno Research, West Grove, PA, USA, 705-485-003 & $1: 500$ \\
\hline Anti-guinea pig IgG Alexa Fluor 594 & Goat & Invitrogen, A11076 & $1: 200$ \\
\hline Anti-guinea pig IgG AMCA & Goat & Jackson Immuno Research, West Grove, PA, USA, 106-156-003 & $1: 500$ \\
\hline Anti-mouse IgG Alexa Fluor 488 & Goat & Invitrogen, A21121 & $1: 200$ \\
\hline Anti-mouse IgG HRP & Goat & Santa Cruz, sc-2005 & $1: 400$ \\
\hline Anti-rabbit IgG Cy3 & Donkey & Jackson Immuno Research, West Grove, PA, USA, 711-165-152 & $1: 500$ \\
\hline Anti-rabbit IgG HRP & Goat & Santa Cruz, sc-2004 & $1: 20000$ \\
\hline anti-rabbit IgG Alexa Fluor 488 & Goat & Invitrogen, A11008 & $1: 200$ \\
\hline
\end{tabular}


(Table 1) was applied with the same buffer as the primary antibody and incubated for $1 \mathrm{~h}$ at RT. Nuclei were counterstained with DAPI, mounted using VECTASHIELDMounting Medium (H-1200, Vector Laboratories, Inc., Burlingame, CA, USA), and observed using a ZEISS Axio Imager.A1 (ZEISS, Jena, Germany). A TUNEL assay was performed according to the manufacturer's instructions (11684817910, Roche Applied Science).

\section{Surface-spread chromatin preparation}

Meiotic cell spreads were prepared as described previously (Peters et al. 1997) with some modifications. Briefly, testes were immersed in PBS, and the retrieved seminiferous tubules were washed and immersed in ice-cold hypotonic extraction buffer (30 mmol/l TRIS, $50 \mathrm{mmol} / \mathrm{l}$ sucrose, $17 \mathrm{mmol} / \mathrm{l}$ trisodium citrate dihydrate, $5 \mathrm{mmol} / \mathrm{I}$ EDTA, $0.5 \mathrm{mmol} / \mathrm{I}$ DTT, and $0.5 \mathrm{mmol} / \mathrm{l}$ phenylmethylsulfonyl fluoride, $\mathrm{pH} 8.2$ ) for $1 \mathrm{~h}$. segments of the tubules were minced in $100 \mathrm{mmol} / \mathrm{l}$ sucrose solution ( $\mathrm{pH} \mathrm{8.2),} \mathrm{and} \mathrm{the} \mathrm{cell} \mathrm{suspension} \mathrm{collected} \mathrm{was} \mathrm{spread}$ onto glass slides, previously immersed in 1\% paraformaldehyde containing $0.15 \%$ Triton X-100; the slides were placed in a moisture box for $2 \mathrm{~h}$. The slides were washed twice for $2 \mathrm{~min}$ with PBS with $0.4 \%$ DRIWEL (Fuji Film, co., Ltd, Tokyo, Japan). After blocking with 5\% skim milk in PBS, the preparation slides were incubated with primary antibodies diluted in the blocking buffer for $3 \mathrm{~h}$ at RT. Secondary antibodies were then incubated for $1 \mathrm{~h}$ at RT. The antibodies used were listed in Table 1 . The slides were then mounted with VECTASHIELD Mounting Medium with DAPI (H-1200, Vector Laboratories, Inc.) and observed using a ZEISS Axio Imager.A1 (ZEISS).

\section{Air-dried chromosome preparation}

The procedure for preparation of chromosomes was as described previously (Fujiwara et al. 2013).

\section{Western blot analysis}

Testes removed from repro57 mutant and control (WT) mice were homogenized in radioimmunoprecipitation assay buffer (RIPA: $150 \mathrm{mM} \mathrm{NaCl}, 10 \mathrm{mM}$ Tris- $\mathrm{HCl}(\mathrm{pH} \mathrm{7.2),} \mathrm{0.1 \%} \mathrm{SDS,}$ $1.0 \%$ Triton $\mathrm{X}-100,1 \%$ deoxycholate, and $5 \mathrm{mM}$ EDTA). The protein extract was applied on 10\% SDS-PAGE and transferred onto Immobilon-P Transfer Membranes (Millipore, Billerica, MA, USA). The membrane was incubated with an antiRNF212 antibody and HRP-conjugated secondary antibody. Subsequently, the same membrane was incubated with antiACTIN antibody after the previous antibodies were removed using Stripping Solution (193-16375; Wako, Osaka, Japan). The antibodies used are listed in Table 1. The membrane was developed using an ECL Advance Western Blotting Detection Kit (RPN2135; Amersham Biosciences) and observed.

\section{Gene expression analysis by RT-PCR}

mRNAs were isolated from the testes of WT and repro57 mutant mice at 10 weeks of age using TRIzol (Invitrogen) following the manufacturer's instructions. Genomic DNA in the RNA sample was digested using DNase I (Takara Bio, Inc., Shiga, Japan), and the RNA solutions were purified by phenol/chloroform treatment. cDNAs were synthesized by RT reaction using Superscripts III reverse transcriptase (Invitrogen) and Oligo dT primer (Invitrogen). RNAs in the cDNA solution were then digested with RNase H (Toyobo, Osaka, Japan). Given evidence for splice variants of Rnf212 (Reynolds et al. 2013) (http://www.ncbi.nlm.nih.gov/nuccore/XM_006535331.1, http://www.ncbi.nIm.nih.gov/nuccore/XM_006535332.1, and http://www.ncbi.nlm.nih.gov/nuccore/XM_001476621.5), the sequences of the variant-specific primers, how they align to the exonic structure, and the PCR cycles are shown in Supplementary Fig. 1., see section on supplementary data given at the end of this article.

\section{Statistical analysis}

Data were expressed as mean \pm s.E.M. The statistical significance of differences in mean values was assessed by Student's $t$-test.

\section{Results}

\section{Infertility and meiotic defects in the testes of repro57 mutant mice}

Both female and male repro57 mutant mice on a congenic $\mathrm{C} 3 \mathrm{H}$ background failed to produce offspring after natural mating with normal heterozygous or WT mice for more than 2 months. Heterozygotes were fully fertile with an average litter size of $6.9 \pm 1.9$ (Table 2). Adult homozygous repro57 mice of both sexes showed no apparent morphological defects in non-reproductive organs, and there was no difference in body weight between the mutant and WT males. However, the testis weight of mutant mice was approximately half that of WT mice (Fig. 1A and B), suggesting germ cell depletion. Histological examination of testes from mutants at 12 weeks of age revealed many degenerating spermatocytes at late prophase stages, with no mature spermatozoa observed in the seminiferous tubules of repro57 mice (Fig. 1D), compared with testes of WT mice (Fig. 1C). A TUNEL assay confirmed increased numbers of apoptotic germ cells in testes of repro57 mice compared with WT mice (Fig. 1E), with cell death specific to metaphase spermatocytes (Fig. 1F).

To determine developmental onset of the phenotype, we examined juvenile WT and repro57 testis histology. At 14 days postpartum (dpp), there were no abnormalities in either WT or repro57 mice (Fig. 2A and B). However, by $21 \mathrm{dpp}$, the seminiferous tubules of WT mice were filled

Table 2 Mating results.

\begin{tabular}{llcc}
\hline Female & Male & $\boldsymbol{n}$ & Number of pups \\
\hline repro57/repro57 & + /repro57 & 4 & 0 \\
+ /repro57 & repro57/repro57 & 3 & 0 \\
+ /repro57 & + /repro57 & 10 & $6.9 \pm 1.9$ \\
\hline
\end{tabular}

$n$, number of mating pairs. Number of pups per litter is expressed as mean \pm s.D. 

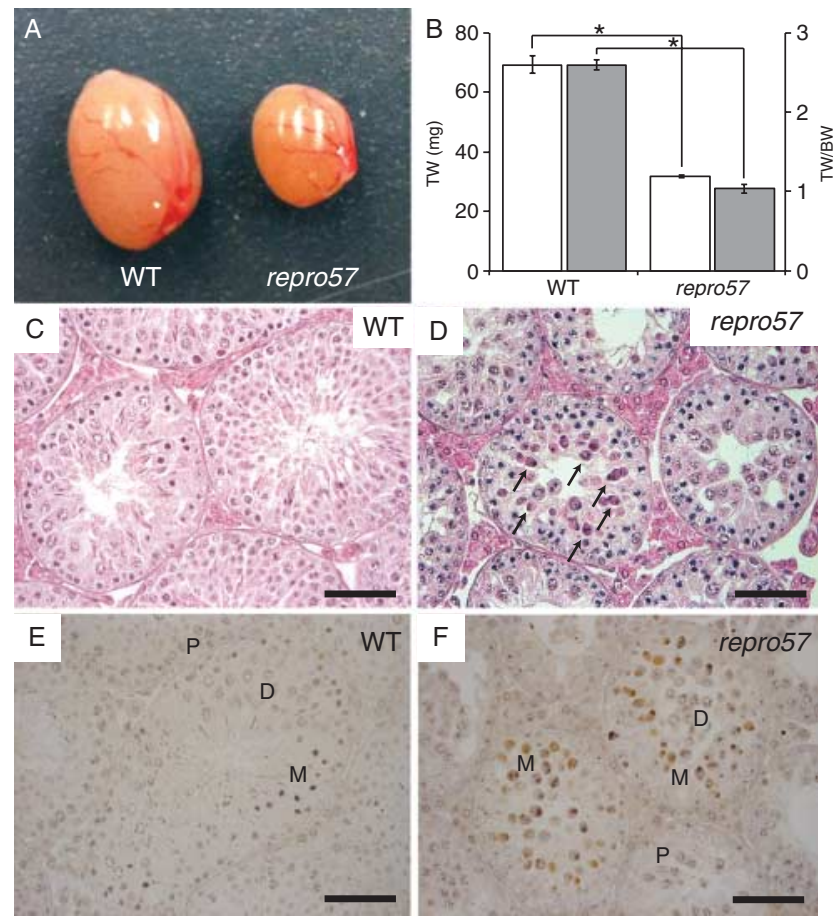

Figure 1 Histology of the testis of WT and repro57 mice. (A) Testes of WT (left) and repro57 (right) mice. (B) The paired testis weights (TW in mg, white bars) and testis-body weight ratios (TW/BW, gray bars) were significantly reduced in repro57 mice $(n=14)$ compared with those of WT mice $(n=8)$. Bars indicate S.E.M; asterisk indicates $P<0.005$. (C and D) HE-stained testis sections show degenerating spermatocytes in the seminiferous tubules of repro57 mice (arrows in D). (E and F) Apoptotic cell death was observed only in metaphase spermatocytes in the testis of repro57 mice. $P$, pachynema; $D$, diplonema; $M$, metaphase spermatocyte. Scale bars; $50 \mu \mathrm{m}$ in $\mathrm{C}, \mathrm{D}, \mathrm{G}$, and $\mathrm{H}$, and $200 \mu \mathrm{m}$ in $\mathrm{E}$ and $\mathrm{F}$.

with round spermatids (Fig. 2C), while those of repro57 mice exhibited degenerating germ cells and no spermatids (Fig. 2D). Reflecting this, testis weights of repro57 mice were significantly lower than that of WT mice at $21 \mathrm{dpp}$ (Fig. 2E). We examined PCNA, which plays an important role in both the DNA synthesis machinery of proliferating mouse spermatogonia (Yazawa et al. 2000) and meiotic progression (Roa et al. 2008). The distribution of PCNA was similar in the testes of WT and repro57 mice (Fig. 2F and G).

\section{Cytological $\mathrm{CO}$ in repro57 spermatocytes}

We assessed marker proteins of meiotic prophase progression to determine abnormalities of meiotic chromosomal dynamics in repro57 mutant testes. The distribution of DMC1, an early recombination repair protein, is similar in WT and repro57 germ cells (Fig. 3A and $B$ ). We determined the pattern of staining for $\gamma \mathrm{H} 2 \mathrm{AX}$ (phosphorylated histone H2AFX), which accumulates at sites of meiotic DNA DSBs in early prophase and, later, is restricted to the $\mathrm{XY}$ body (Fig. 3C). The repro57 mutant spermatocytes exhibited normal distribution of $\gamma \mathrm{H} 2 \mathrm{AX}$ during the pachytene stage (Fig. 3D). However, 30\% of repro57 mutant pachytene spermatocytes showed $\mathrm{XY}$ dissociation (Fig. 3D, $n=260$ cells), while only $3.7 \%$ of WT pachytene spermatocytes exhibited XY dissociation (Fig. 3C, $n=135$ cells).

To identify COs, we immunolabeled for MLH1 foci, which mark COs during mid- to late-pachytene substages and for the testis-specific histone $\mathrm{H} 1$ variant HIST1H1T (herein referred to by its common designation of $\mathrm{H} 1 \mathrm{t}$ ), which is expressed in spermatocytes at post-midpachytene stages (Cobb et al. 1999). H1t-positive spermatocytes in the WT mice contained normal numbers of $\mathrm{MLH} 1$ foci (1-2 foci on each chromosome) (Fig. 4A). However, almost all H1t-positive homozygous
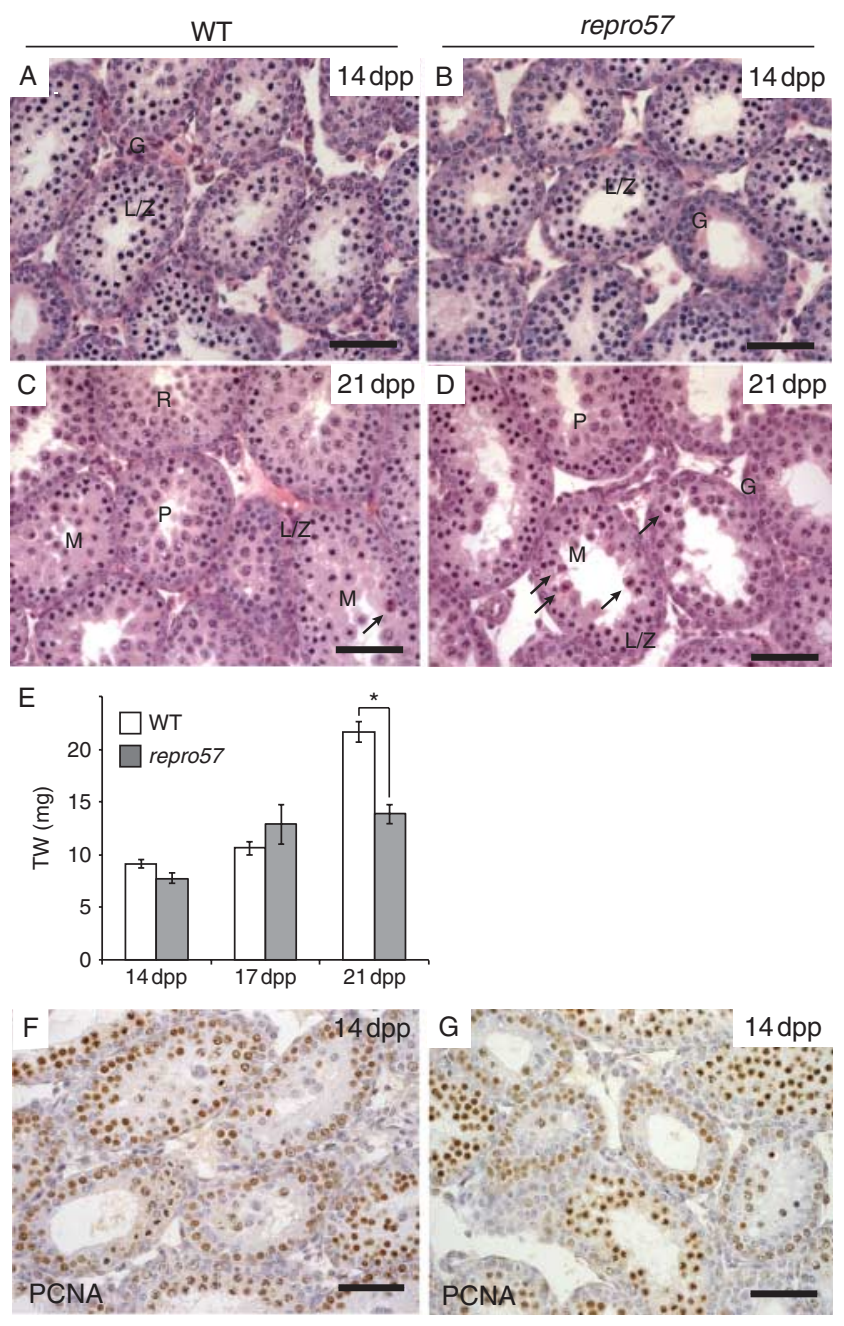

Figure 2 Developmental analysis of testes in WT and repro57 mice. (A, B, C, and D) HE-stained sections of the testis from WT (A and C, $n=5$ and 6 mice at 14 and 21 dpp respectively) and repro57 (B and D, $n=4$ and 3 mice at 14 and $21 \mathrm{dpp}$ respectively) mice during the first wave of spermatogenesis. G, spermatogonia; L/Z, leptonema/zygonema; $P$, pachynema; $R$, round spermatid. Arrows indicate degenerating germ cells. (E) Testis weights for WT and repro57 mice during the peri-pubertal period of development. Bars indicate S.E.M; asterisk indicates $P<0.05$. (F and G) Immunolabeling for PCNA on testis sections from prepubertal WT (A) and repro57 (B) mice at $14 \mathrm{dpp}$. Scale bar, $50 \mu \mathrm{m}$. 


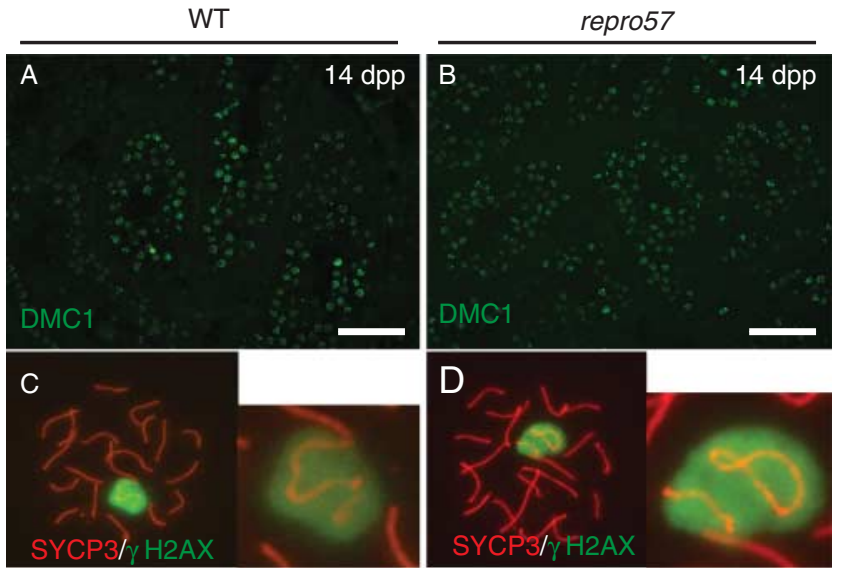

Figure 3 Analysis of DNA damage repair and XY body dynamics in WT and repro57 spermatocytes. (A and B) Sections from 14 dpp testes were immunolabeled for DMC1. Scale bars, $50 \mu \mathrm{m}$. (C and D) Spread spermatocyte nuclei were immunolabeled for SYCP3 (red) and $\gamma \mathrm{H} 2 \mathrm{AX}$ (green), with magnified views of the $X Y$ bodies (insets), revealing $X Y$ chromosome dissociation in repro57 mutant spermatocytes. (original magnification of $\mathrm{E}, \mathrm{F}=\times 1000$ ).

mutant spermatocytes showed complete absence of MLH1 foci (Fig. 4B), although a few had 1-8 MLH1 foci per cell (Fig. 4C). We also scored COs by labeling diplotene spermatocytes for co-localization of SYCP3 and SYCP1 in partially desynapsed diplotene spermatocytes. In contrast to WT spermatocytes, the repro57 mutant spermatocytes lacked SYCP1-SYCP3 co-localization (a mark of CO between homologs).
However, homologs in mutant germ cells were partially associated at centromere (Fig. 4E), as were homologs in WT diplotene spermatocytes (Qiao et al. 2012; Fig. 4D). At diakinesis/metaphase I, all 20 chromosomes exhibited $\mathrm{CO}$ in WT spermatocytes (Fig. 4F), but only a few chromosomes in repro57 mutant spermatocytes exhibited chiasmata (Fig. 4G). Consistent with the lack of chiasmata, there were a significantly increased number of chromosomes (univalents) in mutant spermatocytes compared with WT spermatocytes (Fig. 4H).

\section{A mutation in the Rnf212 gene in repro57 mice}

The repro57 mutation was mapped to $\sim 3 \mathrm{Mb}$ region of Chr. 5 between D5Mit338 and D5Mit117 by linkage analysis using 82 affected F2 male mice (Fig. 5A). This region includes 70 genes; among these, Rnf 212 was a strong candidate gene for harboring the repro57 mutation. Similar to repro57 mutant mice, mice with a targeted gene knockout of Rnf212 exhibit male and female infertility, with male germ cells arrested at late meiotic prophase (Reynolds et al. 2013). High-throughput sequencing of DNA from homozygous repro57 and WT mice revealed a C-to-T transversion at nucleotide 265 of the Rnf 212 mRNA (at $108757107 \mathrm{bp}$ in Chr5, GRCm38.p2), creating a premature stop codon within the coiled-coil domain of RNF212 (Fig. 5B). This mutation was common to all mice with the repro57 phenotype and, moreover, no other exonic mutation was detected within the repro57 region. We assessed expression of Rnf212 transcript variants in
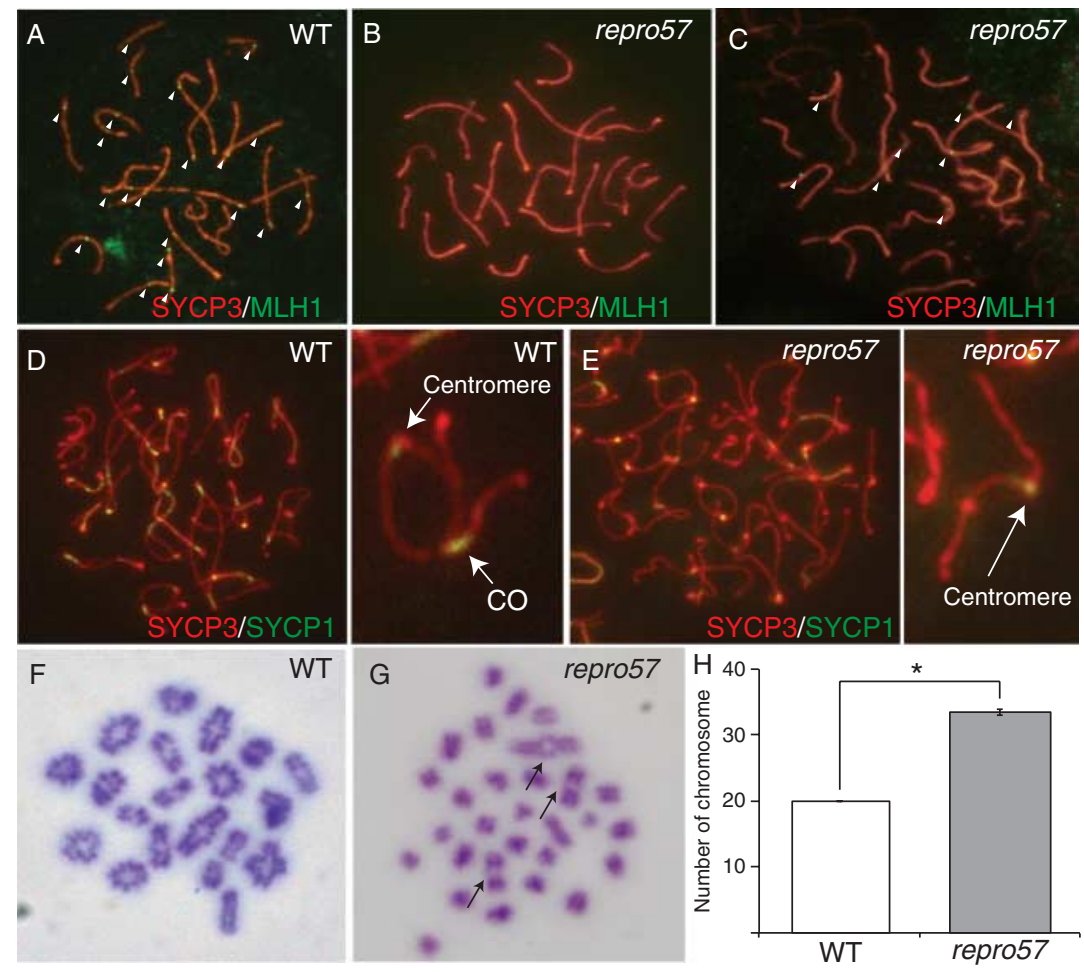

Figure 4 Analysis of crossing-over in WT and repro57 spermatocytes. (A, B, and C) Pachytene spermatocytes from WT (A) and repro57 homozygous mice (B and C) immunolabeled with SYCP3 (red) and MLH1 (green). MLH1 foci are absent in most H1T-positive repro57 spermatocytes (B); however, some mutant spermatocytes exhibited 1-8 weak MLH1 foci (C). Arrowheads indicate MLH1 focus. (D and E) Diplotene spermatocytes immunolabeled with SYCP3 (red) and SYCP1 (green) from (D) WT, with a magnified view of a chromosome with chiasma and (E) repro57, with a magnified view of a chromosome lacking $\mathrm{CO}$. (F, G, and H) Giemsa-stained chromosome of diakinesis/metaphase I spermatocyte from WT (F) and repro57 mice (G). Arrows in G indicate bivalent chromosomes with chiasma, but many lack chiasmata. Original magnification $\times 1000$. (H) The number of chromosomes, including both bivalent and univalent, was compared ( $n=21$ cells for WT and $n=60$ cells for repro57). Bars indicate S.E.M; asterisk indicates $P<0.005$. Data are expressed as mean \pm S.E.M. 

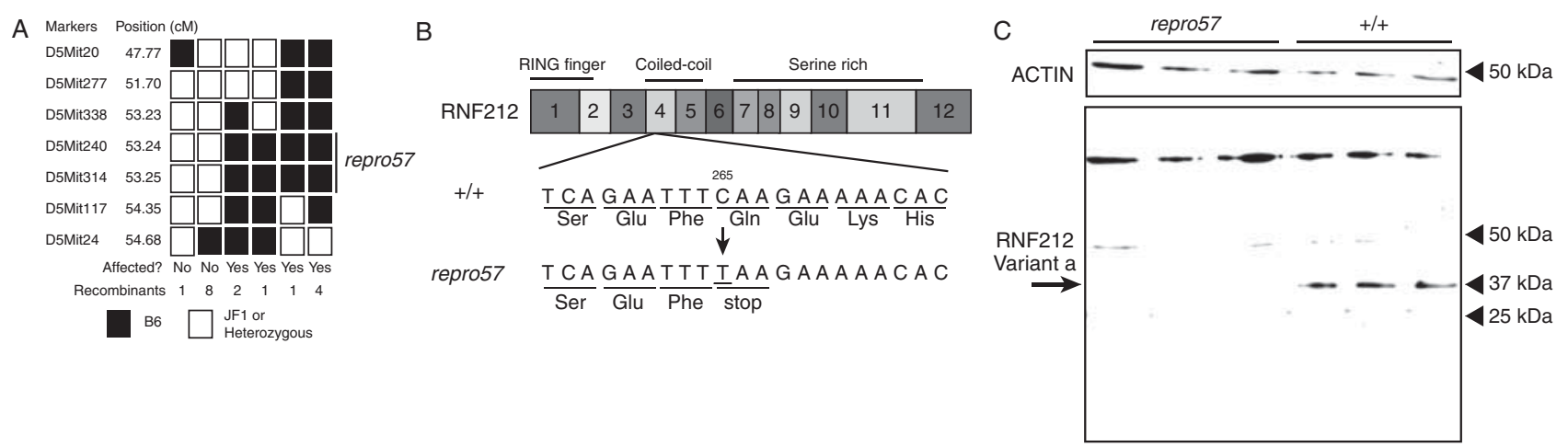

Figure 5 Fine mapping of repro57 mutation and loss of RNF212 expression. (A) Segregation of alleles of seven microsatellite markers in 82 affected mice of the F2 progeny obtained from crosses between + /repro57 and JF1 mice. The positions of the microsatellite markers in the critical region are indicated in $\mathrm{CM}$ according to the mouse genome sequence (NCBI Build 38) and the region that contains the repro57 locus is indicated by a bar. (B) Schematic diagram of the Rnf212 transcript indicating the position of the nonsense mutation in exon 4 of Rnf212. (C) Western blot analysis revealed no expression of RNF212 in the testis of adult repro57 mice, and a single band was detected only in the testes of WT mice. Non-specific bands were detected in the testes of both WT and repro57 mice. ACTIN was used as an internal control.

WT and repro57 testes. Three variants contain the RING finger domain (Reynolds et al. 2013); bioinformatics analysis revealed that $5^{\prime}$ end of two other transcripts contains variant-specific sequences, lacking the RING finger domain (GRCm38.p2, C57BL/6J). RT-PCR analysis of WT and repro57 testis RNAs revealed expression of variants a, b, c, and e in both WT and repro57 mice, while variant $d$ was not detected in either WT or repro57 mice (Supplementary Fig. 1).

To determine whether RNF212 protein is absent in repro57 mice, we performed western blot analysis using whole testis lysates, with a polyclonal antibody raised against the full-length mouse RNF212. In WT testes, a single RNF212 band (correlating to splice variant a) was detected at $\sim 34 \mathrm{kDa}$, while no RNF212, or even a truncated protein, was detected in repro57 mutant testes (Fig. 5C). Non-specific bands at higher molecular sizes were observed in both WT and repro57 lysates.

\section{Discussion}

A moderate-scale unbiased mutagenesis effort coupled with phenotype screening is a powerful tool to create animal models for human disease. We identified the repro57 fertility model by a fertility screen in such an ENU mutagenesis program. We find that there is a nonsense mutation in the Rnf212 gene in repro57 mutant mice. Similar to the Rnf212 knockout phenotype, the repro57 mutant mice exhibit male infertility, arrest of spermatogenesis in meiosis, and defects in cytological markers of recombination and chiasma formation. These observations identify a new allele of the mouse Rnf212 gene, Rnf212 ${ }^{\text {repro57. }}$

\section{A nonsense mutation in Rnf212, encoding a meiotic recombination factor, in repro57 mutants}

Deep sequencing revealed a nonsense mutation in exon 4 of the Rnf212 gene in repro57 mutant. This mutation could truncate the RNF212 protein, ablating the coiled-coil and serine-rich domains. Interestingly, both this study and the previous study (Reynolds et al. 2013) find that only a single RNF212 isoform is expressed in the testis, in spite of evidence for several splicing variants. This could suggest that the variants are not translationally competent.

There is considerable evidence for essential function of RNF212 in meiosis in a variety of species. Rnf212 was shown to be essential for meiotic recombination in Caenorhabditis elegans (Jantsch et al. 2004, Bhalla et al. 2008). In human, variants of RNF212 have been associated with different genome-wide recombination rates (Kong et al. 2008, 2014, Chowdhury et al. 2009). Creation of a targeted mutation, Rnf $212^{\text {tm } 1 \text { Nhtr }}$, revealed that mouse RNF212 was essential for CO formation; the Rnf212 KO spermatocytes lack chiasmata and exhibit depletion of spermatids and mature spermatozoa (Reynolds et al. 2013). These phenotypes of Rnf212 KO mutant mice resemble those of the repro57 mutant mice, providing evidence that the new Rnf212 allele, Rnf $212^{\text {repro57, }}$, is responsible for infertility of the repro57 mice. Final confirmation that the new Rnf $212^{\text {repros }}$ allele is solely causative of the repro57 infertility phenotype awaits a formal genetic complementation test.

\section{Spermatogenic and meiotic defects in repro57 mice}

Many aspects of the spermatogenesis and meiotic phenotype of Rnf212 $2^{\text {repro57 }}$ mutant males reflect those found in Rnf $212 \mathrm{KO}$ males. Even during the juvenile onset of spermatogenesis, degenerating and apoptotic spermatocytes were observed (at $21 \mathrm{dpp}$ ). However, the normal expression pattern of PCNA in the mutant testes suggests that mitotic proliferation and meiotic initiation are normal in the absence of RNF212. Similar to mice with the Rnf212 KO mutation (Reynolds et al. 2013), Rnf212 $2^{\text {repro57 }}$ spermatocytes form the SC and exhibit synapsis. Interestingly, another RNF212 homolog ZHP3 
is also not required for SC formation (Jantsch et al. 2004); however, yeast Zip3 mutants exhibited defective SC formation (Agarwal \& Roeder 2000). Evidence that DNA DSBs are repaired in $R n f 212^{\text {repro57 }}$ spermatocytes includes apparently normal localization of DMC1, which is essential for DNA strand invasion (Moens et al. 2002) and redistribution of $\gamma \mathrm{H} 2 \mathrm{AX}$ by mid-prophase in mutant spermatocytes. However, mutant Rnf212 $2^{\text {repro57 }}$ spermatocytes, similar to Rnf $212 \mathrm{KO}$ spermatocytes, fail to form COs as marked by MLH1 foci, exhibiting lack of chiasmata in most homologous chromosome pairs. Together, observations on mutant spermatocytes for either of the two different Rnf212 alleles suggest that mouse RNF212 plays a role in maturation of CO events in meiotic recombination, but the mechanism is still not clear. Recent evidence suggests that small ubiquitin-like protein (SUMO) plays an important role in assembly and disassembly of SC by regulating protein-protein interaction during meiosis (Watts \& Hoffmann 2011). In this context, it is interesting that the mouse RNF212 mediates SUMOylation, with SUMO modification stabilizing the association of MutS $\gamma$ with nascent $\mathrm{CO}$ intermediates (Reynolds et al. 2013). Human RNF212 and yeast homolog Zip3 contain a RING-finger domain and have ubiquitin-ligating activity for post-translational protein modification (Deshaies \& Joazeiro 2009), and Zip3 has also been reported to be involved in SUMO modification (Perry et al. 2005, Cheng et al. 2006). In this context, it is relevant that the repro57 mutation in the Rnf212 gene may still lead to a protein with an intact RING domain. Loss of RING domain in the targeted Rnf $212 \mathrm{KO}$ allele resulted in absence of MLH1 foci in germ cells (Reynolds et al. 2013), and although the majority of Rnf212 $2^{\text {repro57 }}$ spermatocytes also lacked MLH1 foci, a few had a markedly reduced number of MLH1 foci and chiasmata, suggesting that the Rnf $212^{\text {repro57 }}$ allele could be hypomorphic in this respect. As much of the value of different gene alleles lies in the ability to tease apart protein domain function, further experimental comparisons of the two mutated alleles are warranted.

\section{Rnf212 infertility models}

These results establish the Rnf2 $12^{\text {repro57 }}$ mice as a singlebase change model for male infertility, suggesting the possibility that some human male infertility could be attributed to RNF212 mutation. Although the initial screening of the repro57 mice suggested only male infertility (http://reproductivegenomics.jax.org/mutants/ G1-586-2.html), female repro57 homozygous mice on the congenic $\mathrm{C} 3 \mathrm{H}$ background also exhibited infertility. Interestingly, the $\mathrm{C} 3 \mathrm{H}$ congenic Rnf212 $2^{\text {repro57 }}$ males exhibited a remarkably higher rate of $X Y$ dissociation than the Rnf212 KO mutants on a B6 background (Reynolds et al. 2013). Given also that recent studies have shown genomic variations between subspecies to cause alteration of phenotypes (Keane et al. 2011,
Yang et al. 2011), our findings indicate that phenotypic differences between Rnf212 mutants could be due to not only the allelic difference but also genetic background effects.

In summary, this study identifies a new allele of the mouse Rnf212 gene, Rnf212 repro57, and shows its association with male fertility. These results bolster genetic evidence that RNF212 is essential for CO and chiasma formation during meiosis. As COs promote the accurate segregation of homologs, thereby avoiding aneuploidy, the mouse Rnf212 mutations provide important models for investigating the requirements for accurate gametic chromosome segregation. In humans, chromosome aneuploidy leads to infertility, especially in males (Egozcue et al. 2000), congenital defects such as down syndrome, or high rates of miscarriage due to defective embryo development (Ambartsumyan \& Clark 2008). Thus, genetic models such as these continue to be valuable for human genetic disease.

\section{Supplementary data}

This is linked to the online version of the paper at http://dx.doi. org/10.1530/REP-14-0122.

\section{Declaration of interest}

The authors declare that there is no conflict of interest that could be perceived as prejudicing the impartiality of the research reported.

\section{Funding}

This work was supported by the Japan Society for the Promotion of Science (JSPS), the Strategic Young Researcher Oversea Visits Program for Acceleration Brain Circulation to Y Fujiwara, Sasakawa Scientific Research Grant 22-426 (Japan Science Society) to Y Fujiwara, and the NIH, HD42137 to the Reproductive Genomics Program at The Jackson Laboratory. Research reported in this publication was also partially supported by the National Cancer Institute under award number P30 CA034196 to JAX; the content is solely the responsibility of the authors and not necessarily the official views of the NIH.

\section{Acknowledgements}

The authors thank Lucy Rowe and Mary Barter (The Jackson Laboratory, USA) for preliminary mapping analysis, Drs Bernard de Massy (National Center for Scientific Research, France), Neil Hunter (University of California, Davis, USA), and Shinichiro Chuma (Kyoto University, Japan) for gifts of SYCP3 (guinea pig), RNF212, and SYCP3 (rabbit) antibodies, and Dr Junko Noguchi (National Institute of Agrobiological Sciences, Japan) for comments on histology. They acknowledge the Scientific Services of The Jackson Laboratory for outstanding support. 


\section{References}

Agarwal S \& Roeder GS 2000 Zip3 provides a link between recombination enzymes and synaptonemal complex proteins. Cell 102 245-255. (doi:10.1016/S0092-8674(00)00029-5)

Ambartsumyan G \& Clark AT 2008 Aneuploidy and early human embryo development. Human Molecular Genetics 17 R10-R15. (doi:10.1093/ hmg/ddn170)

Baudat F, Imai Y \& de Massy B 2013 Meiotic recombination in mammals: localization and regulation. Nature Reviews. Genetics 14 794-806. (doi:10.1038/nrg3573)

Bhalla N, Wynne DJ, Jantsch V \& Dernburg AF 2008 ZHP-3 acts at crossovers to couple meiotic recombination with synaptonemal complex disassembly and bivalent formation in Caenorhabditis elegans. PLoS Genetics 4 e1000235. (doi:10.1371/journal.pgen.1000235)

Bolcun-Filas E \& Schimenti JC 2012 Genetics of meiosis and recombination in mice. International Review of Cell and Molecular Biology 298 179-227. (doi:10.1016/B978-0-12-394309-5.00005-5)

Cheng $\mathrm{CH}$, Lo YH, Liang SS, Ti SC, Lin FM, Yeh CH, Huang HY \& Wang TF 2006 SUMO modifications control assembly of synaptonemal complex and polycomplex in meiosis of Saccharomyces cerevisiae. Genes and Development 20 2067-2081. (doi:10.1101/gad.1430406)

Chowdhury R, Bois PR, Feingold E, Sherman SL \& Cheung VG 2009 Genetic analysis of variation in human meiotic recombination. PLoS Genetics 5 e1000648. (doi:10.1371/journal.pgen.1000648)

Cingolani P, Platts A, Wang le L, Coon M, Nguyen T, Wang L, Land SJ, Lu X \& Ruden DM 2012 A program for annotating and predicting the effects of single nucleotide polymorphisms, SnpEff: SNPs in the genome of Drosophila melanogaster strain w1118; iso-2; iso-3. Fly 6 80-92. (doi:10.4161/fly.19695)

Cobb J, Reddy RK, Park C \& Handel MA 1999 Analysis of expression and function of topoisomerase I and II during meiosis in male mice. Molecular Reproduction and Development 46 489-498. (doi:10.1002/ (SICI)1098-2795(199704)46:4<489::AID-MRD7>3.0.CO;2-K)

Cromie GA \& Smith GR 2007 Branching out: meiotic recombination and its regulation. Trends in Cell Biology 17 448-455. (doi:10.1016/j.tcb.2007. 07.007)

DePristo MA, Banks E, Poplin R, Garimella KV, Maguire JR, Hartl C, Philippakis AA, del Angel G, Rivas MA, Hanna M et al. 2011 A framework for variation discovery and genotyping using next-generation DNA sequencing data. Nature Genetics 43 491-498. (doi:10.1038/ng. 806)

Deshaies RJ \& Joazeiro CA 2009 RING domain E3 ubiquitin ligases. Annual Review of Biochemistry 78 399-434. (doi:10.1146/annurev.biochem.78. 101807.093809)

Egozcue S, Blanco J, Vendrell JM, Garcia F, Veiga A, Aran B, Barri PN, Vidal F \& Egozcue J 2000 Human male infertility: chromosome anomalies, meiotic disorders, abnormal spermatozoa and recurrent abortion. Human Reproduction Update 6 93-105. (doi:10.1093/humupd/6.1.93)

Fujiwara Y, Ogonuki N, Inoue K, Ogura A, Handel MA, Noguchi J \& Kunieda T 2013 t-SNARE Syntaxin2 (STX2) is implicated in intracellular transport of sulfoglycolipids during meiotic prophase in mouse spermatogenesis. Biology of Reproduction 88 141. (doi:10.1095/ biolreprod.112.107110)

Handel MA \& Schimenti JC 2010 Genetics of mammalian meiosis: regulation, dynamics and impact on fertility. Nature Reviews. Genetics 11 124-136. (doi:10.1038/nrg2723)

Handel MA, Lessard C, Reinholdt L, Schimenti J \& Eppig JJ 2006 Mutagenesis as an unbiased approach to identify novel contraceptive targets. Molecular and Cellular Endocrinology 250 201-205. (doi:10. 1016/j.mce.2005.12.046)

Jantsch V, Pasierbek P, Mueller MM, Schweizer D, Jantsch M \& Loidl J 2004 Targeted gene knockout reveals a role in meiotic recombination for ZHP-3, a Zip3-related protein in Caenorhabditis elegans. Molecular and Cellular Biology 24 7998-8006. (doi:10.1128/MCB.24.18.7998-8006.2004)

Keane TM, Goodstadt L, Danecek P, White MA, Wong K, Yalcin B, Heger A, Agam A, Slater G, Goodson M et al. 2011 Mouse genomic variation and its effect on phenotypes and gene regulation. Nature 477 289-294. (doi:10.1038/nature10413)

Kong A, Thorleifsson G, Stefansson H, Masson G, Helgason A, Gudbjartsson DF, Jonsdottir GM, Gudjonsson SA, Sverrisson S,
Thorlacius T et al. 2008 Sequence variants in the RNF212 gene associate with genome-wide recombination rate. Science 319 1398-1401. (doi:10.1126/science.1152422)

Kong A, Thorleifsson G, Frigge ML, Masson G, Gudbjartsson DF, Villemoes R, Magnusdottir E, Olafsdottir SB, Thorsteinsdottir U \& Stefansson K 2014 Common and low-frequency variants associated with genome-wide recombination rate. Nature Genetics 46 11-16. (doi:10.1038/ng.2833)

Lessard C, Pendola JK, Hartford SA, Schimenti JC, Handel MA \& Eppig JJ 2004 New mouse genetic models for human contraceptive development. Cytogenetic and Genome Research 105 222-227. (doi:10.1159/ 000078192)

Li H \& Durbin R 2009 Fast and accurate short read alignment with BurrowsWheeler transform. Bioinformatics 25 1754-1760. (doi:10.1093/ bioinformatics/btp324)

McKenna A, Hanna M, Banks E, Sivachenko A, Cibulskis K, Kernytsky A, Garimella K, Altshuler D, Gabriel S, Daly M et al. 2010 The Genome Analysis Toolkit: a MapReduce framework for analyzing next-generation DNA sequencing data. Genome Research 20 1297-1303. (doi:10.1101/ gr.107524.110)

Moens PB, Kolas NK, Tarsounas M, Marcon E, Cohen PE \& Spyropoulos B 2002 The time course and chromosomal localization of recombinationrelated proteins at meiosis in the mouse are compatible with models that can resolve the early DNA-DNA interactions without reciprocal recombination. Journal of Cell Science 115 1611-1622.

Patel RK \& Jain M 2012 NGS QC Toolkit: a toolkit for quality control of next generation sequencing data. PLOS ONE 7 e30619. (doi:10.1371/journal. pone.0030619)

Perry J, Kleckner N \& Borner GV 2005 Bioinformatic analyses implicate the collaborating meiotic crossover/chiasma proteins Zip2, Zip3, and Spo22/Zip4 in ubiquitin labeling. PNAS 102 17594-17599. (doi:10. 1073/pnas.0508581102)

Peters AH, Plug AW, van Vugt MJ \& de Boer P 1997 A drying-down technique for the spreading of mammalian meiocytes from the male and female germline. Chromosome Research 5 66-68. (doi:10.1023/ A:1018445520117)

Phadnis N, Hyppa RW \& Smith GR 2011 New and old ways to control meiotic recombination. Trends in Genetics 27 411-421. (doi:10.1016/j. tig.2011.06.007)

Qiao H, Chen JK, Reynolds A, Hoog C, Paddy M \& Hunter N 2012 Interplay between synaptonemal complex, homologous recombination, and centromeres during mammalian meiosis. PLoS Genetics 8 e1002790. (doi:10.1371/journal.pgen.1002790)

Reynolds A, Qiao H, Yang Y, Chen JK, Jackson N, Biswas K, Holloway JK, Baudat F, de Massy B, Wang J et al. 2013 RNF212 is a dosage-sensitive regulator of crossing-over during mammalian meiosis. Nature Genetics 45 269-278. (doi:10.1038/ng.2541)

Roa S, Avdievich E, Peled JU, Maccarthy T, Werling U, Kuang FL, Kan R, Zhao C, Bergman A, Cohen PE et al. 2008 Ubiquitylated PCNA plays a role in somatic hypermutation and class-switch recombination and is required for meiotic progression. PNAS 105 16248-16253. (doi:10. 1073/pnas.0808182105)

Watts FZ \& Hoffmann E 2011 SUMO meets meiosis: an encounter at the synaptonemal complex: SUMO chains and sumoylated proteins suggest that heterogeneous and complex interactions lie at the centre of the synaptonemal complex. BioEssays 33 529-537. (doi:10.1002/bies. 201100002)

Yang H, Wang JR, Didion JP, Buus RJ, Bell TA, Welsh CE, Bonhomme F, Yu AH, Nachman MW, Pialek J et al. 2011 Subspecific origin and haplotype diversity in the laboratory mouse. Nature Genetics 43 648-655. (doi:10.1038/ng.847)

Yazawa T, Yamamoto T, Nakayama Y, Hamada S \& Abe S 2000 Conversion from mitosis to meiosis: morphology and expression of proliferating cell nuclear antigen (PCNA) and Dmc1 during newt spermatogenesis. Development, Growth \& Differentiation 42 603-611. (doi:10.1046/ j.1440-169x.2000.00544.x)

Received 3 March 2014

First decision 3 April 2014

Revised manuscript received 7 October 2014

Accepted 23 October 2014 